\title{
Determinación de la Huella del Carbono mediante el Método Compuesto de las Cuentas Contables (MC3) para una Empresa Vitivinícola en Chile
}

\author{
Rafael Quezada ${ }^{(1)}$, Tailin Hsieh ${ }^{(1)}$ y José O. Valderrama ${ }^{(2,3)}$ \\ (1) Univ. de La Serena, Fac. de Ingeniería, Dpto. de Ing. Industrial, Casilla 554, La Serena-Chile \\ (2) Univ. de La Serena, Fac. de Ingeniería, Dpto. de Ing. Mecánica, Casilla 554, La Serena-Chile \\ (3) Centro de Información Tecnológica (CIT), Casilla 724, La Serena-Chile
}

Recibido Feb. 04, 2013; Aceptado Mar. 15, 2013; Versión final recibida Abr. 10, 2013

\begin{abstract}
Resumen
Se determina la huella del carbono $(\mathrm{HdC})$ en una empresa vitivinícola mediante el Método Compuesto de las Cuentas Contables versión 2.0 (MC3) considerado uno de los procedimientos más simples de implementar en una empresa. Se discuten aspectos relacionados con la adopción por parte de la empresa del concepto de la $\mathrm{HdC}$, como un elemento claro sobre cuán amigable con el medio ambiente ha sido el proceso de elaboración de sus productos. Hoy en día, y en el futuro cercano, el contar con una medición adecuada de la HdC constituye un factor de competitividad importante, en especial en el mercado internacional que inevitablemente impondrá restricciones basadas en producción sustentable. Se aplicó el método MC3 a la empresa vitivinícola denominada Viña Modelo y se obtuvo como resultado una HdC de $0.2 \mathrm{Kg}$. de $\mathrm{CO}_{2}$ por botella de vino producida. Este resultado es inferior a otras mediciones similares realizadas en otros países del mundo y disponibles en la literatura, aspecto que es también analizado.
\end{abstract}

Palabras clave: huella del carbono, método MC3, industria vitivinícola, gases efecto invernadero, calentamiento global

\section{Determination of the Carbon Footprint using the Method Composed of Financial Accounts (MC3) for a Wine Company in Chile}

\begin{abstract}
The carbon footprint (CF) in a winery company is determined using the Method Composed of Financial Accounts (MC3), considered to be one of the simplest procedures to be implemented in a company. Aspects related to the adoption of the CF concept by the company, as a clear element to determine how environmentally friendly has been the product manufacture, are discussed. Nowadays, and in the near future, having an appropriate determination of the CF is an important factor of competitiveness, especially in the international market that will inevitably impose restrictions based on sustainable production. The MC3 method was applied to the company Viña Modelo and the CF found was 2 tons of $\mathrm{CO}_{2}$ per bottle of wine. This result is lower than values reported in the literature for other companies around the world, aspect that is also analyzed.
\end{abstract}

Keywords: carbon footprints, MC3 method, wineries, greenhouse gases, global warming 


\section{INTRODUCCIÓN}

Todos los bienes que se producen y consumen y los servicios que se prestan y reciben tienen algún impacto sobre el medio ambiente y producen gases contaminantes durante su producción, almacenamiento, transporte, uso y disposición final. La importancia de estimar y reducir las emisiones de algunos de estos gases se encuentra en su aporte al llamado efecto invernadero, razón por la que se denominan gases efecto invernadero (GEI). Este efecto se manifiesta por el calentamiento de la atmósfera debido a la formación de una capa con los gases contaminantes en la atmósfera media (a unos $80.000 \mathrm{~m}$. sobre la superficie de la tierra). La energía que llega del sol está formada por ondas de frecuencias altas que traspasan la atmósfera sin mucha resistencia. Sin embargo, la energía remitida desde la tierra hacia el exterior está formada por ondas de frecuencias más bajas que son absorbidas por la capa de GEI. En forma simple, el efecto invernadero provoca que la energía que llega a la tierra sea devuelta más lentamente, por lo que es mantenida más tiempo junto a la superficie elevando la temperatura (Bolin et al., 1986), fenómeno que en términos genéricos se denomina calentamiento global.

Existe una serie de GEI que contribuyen en mayor o menor medida al calentamiento global. Por ello se ha definido una escala de potencial de calentamiento global (PCG) que depende del poder de radiación y la permanencia promedio del gas en la atmósfera. Este potencial se calcula matemáticamente y se expresa en relación con el PCG del dióxido de carbono, al que se le asigna el valor 1. Así el metano por ejemplo tiene un PCG de 25 y el Freon 12 de 11 mil 1 (BSI, 2008). Aparte de ser un problema ambiental, el calentamiento global constituye también un problema de desarrollo, con profundos impactos en la sociedad, la economía y los ecosistemas. En este contexto ha surgido el concepto de huella del carbono ( $\mathrm{HdC})$ que se ha convertido en pocos años en un lema en el debate público sobre el calentamiento global y el cambio climático, atrayendo la atención de los consumidores, negocios, gobiernos, organizaciones no-gubernamentales e instituciones internacionales (Peters y Hertwich, 2008). La HdC representa la cantidad de gases de efecto invernadero emitidos a la atmósfera derivados de las actividades de producción o consumo de bienes y servicios, y es considerada una de las más importantes herramientas para cuantificar las emisiones de dichos gases (Espíndola y Valderrama, 2012a).

En el caso de los países en vías de desarrollo, como ocurre con la mayoría de los países latinoamericanos, un patrón exportador más acorde con las aspiraciones de desarrollo sostenible y menos vulnerable a las exigencias climáticas, exige a los sectores productivos realizar avances inmediatos en los procesos de cuantificación de sus emisiones y de disminución de los efectos climáticos, con el fin de resguardar su actual posición competitiva (Espíndola y Valderrama, 2012b). La industria vitivinícola no ha estado ajena al problema y hay importantes avances en Italia, Francia y Australia (Bosco et al., 2011; Waye, 2008). Varias empresas vitivinícolas chilenas han decidido incorporarse a esta tendencia y han iniciado el proceso cuantificando los diversos factores que afectan la HdC para determinar la situación actual e innovar en el futuro.

Actualmente existen cuatro métodos principales presentados en la literatura para el cálculo de la HdC en empresas, organizaciones, servicios, procesos y productos. Estos métodos utilizan un acercamiento muy similar para la obtención de datos, como es el análisis de ciclo de vida del producto. Una vez que se esquematiza el ciclo de vida del producto, desde que se extraen las materias primas necesarias para su fabricación hasta el lugar de su disposición final, cada método usa un enfoque diferente. La primera diferencia tiene lugar en la limitación del alcance de la empresa, es decir hasta dónde las emisiones de otros procesos influyen en el proceso en estudio. Una vez que se tiene el proceso bien delimitado, se extraen datos diferentes del proceso, para cada método de determinación. La Tabla 1 resume algunas características de los cuatro métodos principales disponibles para cuantificar la HdC denominados GHG Protocol, Bilan Carbone, PAS 2050 y MC3.

Tabla 1: Métodos de determinación de la HdC (adaptada de Valderrama et al, 2011)

\begin{tabular}{|c|c|c|c|}
\hline Método & Enfoque & Referencia/País & Unidad \\
\hline $\begin{array}{l}\text { Protocolo de Gases Efecto Invernadero } \\
\text { (GEI Protocol) }\end{array}$ & Corporativo & $\begin{array}{l}\text { GEI Protocol (2001), } \\
\text { Multinacional }\end{array}$ & Ton $\mathrm{CO}_{2}$ /año; Ton $\mathrm{CO}_{2}$ /unid \\
\hline Balance de Carbono (Bilan Carbone) & Corporativo & ABC (2012), Francia & Ton $\mathrm{CO}_{2}$ /año; Ton $\mathrm{CO}_{2}$ /unid \\
\hline $\begin{array}{l}\text { Especificaciones Públicamente } \\
\text { Disponibles (PAS 2050) }\end{array}$ & Producto & BSI (2008), UK & Ton $\mathrm{CO}_{2} /$ unid. \\
\hline $\begin{array}{l}\text { Método Compuesto de las Cuentas } \\
\text { Contables (MC3) }\end{array}$ & $\begin{array}{l}\text { Corporativo y } \\
\text { Producto }\end{array}$ & $\begin{array}{l}\text { Doménech (2004) } \\
\text { España }\end{array}$ & $\begin{array}{l}\text { Ton } \mathrm{CO}_{2} / \text { año /unid } \\
\text { Hectárea Global }\end{array}$ \\
\hline
\end{tabular}


La preocupación por el impacto ambiental en la industria vitivinícola, en particular por emisiones de GEI, ha aumentado en los últimos años y se ha vuelto un elemento central dentro de su gestión (Borregaard et al., 2009). La práctica de la vitivinicultura es altamente dependiente de las condiciones climáticas y cualquier cambio en las estaciones tales como su duración, las temperaturas máximas, mínimas o medias, o la ocurrencia de heladas puede tener un gran impacto en las regiones vitivinícolas del mundo. Dado también que la industria vitivinícola tiene grandes necesidades energéticas, la tendencia actual es hacia la adopción de tecnologías limpias y con menor consumo energético (Smyth y Russell, 2009). La Tabla 2 muestra la variedad de temas de estudio alrededor del concepto de la HdC, demostrando el creciente interés sobre los impactos ambientales de la industria vitivinícola, en especial por parte de los países exportadores e importadores de vino en el mundo.

Tabla 2: Algunos estudios relacionados con la $\mathrm{HdC}$ en la industria vitivinícola mundial

\begin{tabular}{|l|l|l|}
\hline País & Aplicación & Referencia \\
\hline \multirow{4}{*}{ Italia } & $\begin{array}{l}\text { Se estima la huella ecológica y se compara la de un vino orgánico con uno } \\
\text { convencional }\end{array}$ & Niccolucci et al., 2008 \\
\cline { 2 - 3 } & Se aplica el análisis del ciclo de vida a la cadena de suministro del vino & Cichelli et al., 2012 \\
\cline { 2 - 3 } & Se comparan las emisiones de GEl de 4 vinos de Maremma en Italia & Bosco et al., 2011 \\
\hline \multirow{2}{*}{ Australia } & $\begin{array}{l}\text { Se discuten los impactos de la estimación de la HdC en la industria } \\
\text { vitivinícola australiana }\end{array}$ & Waye, 2008 \\
\cline { 2 - 3 } & $\begin{array}{l}\text { Se estudia la percepción de los clientes de los vinos convencionales versus } \\
\text { los eco-amigables en Australia }\end{array}$ & Sirieix y Remaud, 2010 \\
\hline \multirow{2}{*}{ EEUU } & $\begin{array}{l}\text { Se analizan los efectos en las emisiones de GEl al usar diferentes } \\
\text { empaques para vino }\end{array}$ & Thompson, 2010 \\
\hline $\begin{array}{l}\text { Reino } \\
\text { Unido }\end{array}$ & $\begin{array}{l}\text { Se analiza el uso de energía en la vitivinicultura y la posibilidad de utilizar } \\
\text { tecnologías renovables para la generación eléctrica }\end{array}$ & Smyth y Russell, 2009 \\
\hline \multirow{2}{*}{$\begin{array}{l}\text { Nueva } \\
\text { Zelanda }\end{array}$} & $\begin{array}{l}\text { Se estudia el rol de la sustentabilidad en la cadena de valor en la industria } \\
\text { del vino Neozelandés }\end{array}$ & Flint y Golicic, 2009 \\
\cline { 2 - 3 } & Se examinan las prácticas sostenibles de un pequeño productor de vino & Thompson y Forbes, 2011 \\
\hline Chile & $\begin{array}{l}\text { Se propone la unidad de vino equivalente energético para la medición de } \\
\text { Eficiencia Energética }\end{array}$ & Borregaard et al., 2009 \\
\hline
\end{tabular}

De los cuatro métodos descritos en la Tabla 1, se ha usado el Método Compuesto de las Cuentas Contables versión 2.0 (de ahora en adelante abreviado simplemente como MC3) por sus varias características amigables. En especial destaca la fácil disponibilidad de los datos requeridos para el cálculo de la HdC, como son la contabilidad y los balances regulares de la empresa.

\section{EL MÉTODO COMPUESTO DE LAS CUENTAS CONTABLES (MC3)}

La versión 2.0 del método $\mathrm{MC}$, consistente en una Planilla de cálculo con 20 hojas, incluye una modificación de las secciones con respecto al método original y presenta nuevas categorías; esto es, nuevos posibles aportes a la HdC. La nueva versión también considera la compra de todo tipo de bienes y servicios, la ocupación del espacio y la generación de residuos, permitiendo calcular la HdC de cualquier organización (Doménech, 2010). La primera hoja de la planilla incluye una guía para la utilización de la hoja de cálculo donde explica la forma en que se consideran los distintos aportes a la HdC. La segunda hoja de la planilla incluye una serie de datos básicos que deben ser actualizados para el caso de estudio. Entre éstos están algunos precios variables (en especial de combustibles), datos para indicadores de sostenibilidad (como la renta anual por ejemplo) y factores de equivalencia, de rendimiento y de absorción de $\mathrm{CO}_{2}$ para terrenos y aguas. Esta información básica se detalla en la Tabla 3.

La tercera hoja de la planilla Excel, llamada HC, es la hoja principal para el ingreso de información. Esta contiene en sus filas el consumo de bienes y servicios de las principales categorías de insumos y materiales que requiere el caso en estudio. El resto de las hojas incluye variada información relacionada con los distintos factores incluidos en el cálculo de la HdC. Por ejemplo, datos arancelarios, matriz de impuestos, datos sobre residuos y vertidos, matriz de gases contaminantes, descripción de los tipos de servicios y varios factores de conversión y equivalencia. Algunas de estas hojas deben ser intervenidas para ingresar datos específicos o actualizar otros, como se describe más adelante. En una hoja aparte llamada "out" aparecen los resultados finales expresados de diversas formas, entre ellas la $\mathrm{HdC}$ en ton. de $\mathrm{CO}_{2} / a n ̃ o$. 
Tabla 3: Datos Básicos de Entrada incluidos en la segunda hoja de la Planilla del método MC3 (tomados de http://bit.ly/10w3Pvx

\begin{tabular}{|c|c|c|}
\hline \multicolumn{2}{|l|}{ Precios Variables (sin IVA) } & \multirow[b]{2}{*}{ Año } \\
\hline & Precio & \\
\hline Precio KWh & 0.115 & 2010 \\
\hline Precio gas ciudad $\left(€ / \mathrm{m}^{3}\right)$ & 0.388 & 2005 \\
\hline Precio gasolina $95(€ / l)$ & 0.88 & 2009 \\
\hline Precio gasolina $98(€ / l)$ & 0.95 & 2009 \\
\hline Precio gasoil A (€/l) & 0.81 & 2009 \\
\hline Precio gasoil $B(€ / l)$ & 0.42 & 2009 \\
\hline Precio gasoil C $(€ / l)$ & 0.42 & 2009 \\
\hline Fuel $(€ / t)$ & 130 & 2004 \\
\hline Biodiesel 100\% (€/l) & 0.564 & 2008 \\
\hline Coste del agua (sin IVA) $\left(€ / \mathrm{m}^{3}\right)$ & 1.536 & 2009 \\
\hline
\end{tabular}

\begin{tabular}{|l|c|c|}
\hline \multicolumn{3}{|c|}{ Datos Para Indicadores De Sostenibilidad } \\
\hline & Valor & Año \\
\hline Valor del euro en dólares & 1.182 & dic-05 \\
\hline Renta global 2000 & 8938.4 & 2000 \\
\hline Renta global 2005 & 8700.8 & 2005 \\
\hline
\end{tabular}

\begin{tabular}{|l|c|c|c|c|c|c|}
\hline & \multicolumn{2}{|r|}{ Factores De } & \multicolumn{2}{r|}{ Factores De } & \multicolumn{2}{|r|}{ Factores De } \\
\hline & \multicolumn{2}{|c|}{ Equivalencia } & \multicolumn{2}{r|}{ Rendimiento } & \multicolumn{2}{|c|}{ Absorción (TCO $/ \mathrm{Ha})$} \\
\hline & Tasa & Fuente & Tasa & Fuente & Tasa & Fuente \\
\hline Bosques para CO2 & 1.33 & GFN & & & 3.67 & IPCC (2001) \\
\hline Superficie cultivable & 2.64 & GFN & 0.75 & GFN & 1.98 & ECCP (2004) \\
\hline Pastos & 0.50 & GFN & 1.21 & GFN & 0.84 & comentario \\
\hline Bosques & 1.33 & GFN & 0.64 & GFN & 3.67 & IPCC (2001) \\
\hline Superficie construida & 2.64 & GFN & 0.75 & GFN & 1.98 & \\
\hline Mar & 0.40 & GFN & 1.07 & GFN & 0.24 & comentario \\
\hline Aguas continentales & 0.40 & GFN & 1.00 & GFN & 0.24 & comentario \\
\hline
\end{tabular}

La información necesaria para estimar la HdC es obtenida principalmente de documentos contables como el balance general de ocho columnas y el libro de costos, lo que permite una clara delimitación de las actividades asociadas a la organización. El procedimiento de cálculo para el método MC3 considera las nueve categorías principales mostradas en la Tabla 4, aunque en un caso particular de aplicación algunas categorías no se consideran porque no existen, como se explica más adelante para el caso especial de la Viña Modelo.

Las categorías de emisiones directas e indirectas (puntos 1 y 2 en la Tabla 4) se centran en las emisiones generadas por el uso de combustible, el cual se clasifica como se muestra en la Tabla 5. En el caso de Viña Modelo se usa principalmente gasolina tipo A (diésel) en vehículos y en la generación de electricidad para actividades nocturnas. Se considera como emisiones indirectas todas aquellas fuentes de energía que aportan a la generación de la electricidad. Para el cálculo de la huella de esta categoría, se hace necesario ajustar el mix energético a la situación del país (Chile en este caso) para ser incorporado en la planilla. En la Tabla 6 se presenta el mix eléctrico chileno mostrando los tipos de generación y el porcentaje de aporte. Para el ingreso de datos de esta categoría se toma el gasto total en electricidad, y según los porcentajes mostrados en la Tabla 6 se realiza un cálculo simple para determinar el gasto relacionado a cada tipo de generación eléctrica, para luego ingresarlos en las celdas correspondientes. 
Tabla 4: Fuentes de emisiones consideradas en MC3 (Tomada de Doménech et al, 2010)

\begin{tabular}{|l|l|}
\hline Categorías de Consumo & Subcategorías \\
\hline 1. Emisiones Directas & 1.1 Combustible \\
& 1.2 Otras emisiones directas \\
\hline 2. Emisiones Indirectas & 2.1 Electricidad \\
& 2.2 Otras emisiones indirectas \\
\hline 3. Materiales & 3.1. Materiales de flujo (mercancías) \\
& 3.2. Materiales no amortizables \\
& 3.3. Materiales amortizables (genéricos) \\
& 3.4. Materiales amortizables (obras) \\
& 3.5. Uso infraestructuras públicas \\
\hline 4. Servicios y Contratas & 4.1. Servicios con baja movilidad \\
& 4.2. Servicios con alta movilidad \\
& 4.3. Servicios de transporte de personas \\
& 4.4. Servicios de transporte de mercancías \\
& 4.5. Uso de infraestructuras públicas \\
\hline 5. Recursos Agropecuarios y & 5.1. Vestuario y manufacturas \\
Pesqueros & 5.2. Productos agropecuarios \\
& 5.3. Servicios de restaurante \\
\hline 6. Recursos Forestales & (no hay subcategorías) \\
\hline 7. Huella Hídrica & 7.1. Consumo de agua potable \\
& 7.2. Consumo de agua no potable \\
\hline 8. Uso Del Suelo & 8.1.Sobre tierra firme \\
& 8.2. Sobre agua \\
\hline 9. Residuos, Vertidos y Emisiones & 9.1. Residuos no peligrosos \\
& 9.2. Residuos peligrosos \\
& 9.3. Residuos radiactivos \\
9.4. Vertidos en efluentes \\
9.5. Emisiones \\
\hline \multicolumn{1}{|c|}{9.5 .1$. Gases GEl Protocolo Kyoto } \\
& 9.5.3. Otros GEl o precursores \\
& \\
\hline
\end{tabular}

Tabla 5: Tipos de combustible (Tomada de Doménech et al, 2010)

\begin{tabular}{|l|c|l|c|}
\hline Combustible & Unidad & Combustible & Unidad \\
\hline Carbón (combustión) & {$[\$]$} & Gasoil A & {$[\mathrm{l}]$} \\
\hline Leña (combustión) & {$[\$]$} & Gasoil B & {$[\mathrm{l}]$} \\
\hline Biomasa de madera & {$[\$]$} & Gasoil C & {$[\mathrm{l}]$} \\
\hline Biomasa (no madera) & {$[\$]$} & Fuel & {$[\mathrm{l}]$} \\
\hline Gas natural & {$\left[\mathrm{m}^{3}\right]$} & Biodiesel 100\% (de cultivos) & {$[\mathrm{l}]$} \\
\hline Gasolina 95 & {$[\mathrm{l}]$} & Biodiesel 100\% (de aceites) & {$[\mathrm{l}]$} \\
\hline Gasolina 98 & {$[\mathrm{l}]$} & Bioetanol 100\% & {$[\mathrm{l}]$} \\
\hline
\end{tabular}

Tabla 6: Mix eléctrico nacional según tipo de generación (Tomada de Arias y Arruez, 2011)

\begin{tabular}{|l|r|}
\hline Tipo de Generación & MWh (\%) \\
\hline Térmica Carbón & 13.0 \\
\hline Ciclo Combin. Gas & 37.1 \\
\hline Nuclear & 0 \\
\hline Hidráulica & 49.2 \\
\hline Mini-Hidráulica & 0 \\
\hline Co-generación & 0 \\
\hline Eólica & 0.7 \\
\hline Fotovoltaica & 0 \\
\hline Solar Térmica & 0 \\
\hline Biomasa & 0.1 \\
\hline Residuos & 0 \\
\hline
\end{tabular}


La categoría materiales (punto 3 en la Tabla 4) se subdivide en cinco subcategorías. Los materiales de flujo, los materiales no amortizables, los materiales amortizables genéricos y en obras, y el uso de la infraestructura pública. En el caso de estudio, se destacan los gastos en los derivados del vidrio y abono, realizando una previa conversión en la hoja adicional llamada "matriz de obras". Esta posibilita la introducción e importe en euros $(€)$, extraídos de las cuentas contables. Para el caso en estudio, los valores fueron ingresados en pesos chilenos (\$) usando el valor referencial a mayo de 2011 ( $1 €=\$ 672)$.

La categoría de Servicios y Contratas (punto 4 en la Tabla 4) contempla cinco subcategorías. Los servicios de alta, baja movilidad, poca o nula movilidad, de transporte de personas, mercancías y eso de infraestructuras públicas. Para los servicios de transporte y mercancías se utiliza la intensidad energética en giga joules por pasajero y kilómetros recorridos por cada tonelada de mercancía, para luego hacer la conversión de energía a carbono, utilizando los mismos factores de emisión usados para el resto de las categorías. En la segunda versión del MC3 usada en este trabajo se ha incluido la huella del uso de infraestructuras y otros bienes públicos, por medio del impuesto al valor agregado declarado, los impuestos pagados y las multas y sanciones. Para la obtención de la HdC de esta categoría, fue necesario clasificar la información extraída de las cuentas contables en las subcategorías que presenta el método, destacándose los impuestos incurridos por el uso de infraestructuras públicas, las de baja movilidad y la de transporte de personas, siendo de menor importancia los servicios de alta movilidad, teniendo en cuenta las unidades exigidas en cada tipo de servicio.

Con la huella de los recursos agrícolas y pesqueros, en conjunto con la forestal y el uso del suelo (puntos 5 , 6 y 8 en la Tabla 4), se entra a un grupo de categorías en las cuales la ocupación del suelo es de gran importancia por su aporte a la HdC final. Para el caso de estudio, las cuentas se centran particularmente en el uso de productos agrícolas para el vestuario y manufactura, los productos agropecuarios y los servicios de restaurante para los alimentos de consumo diario, y los distintos productos forestales, tales como estibas, pilotes, paneles, mobiliario a base de madera y productos editoriales. Cabe destacar la importancia de esta última sección, debido principalmente al fuerte consumo de productos madereros en el proceso agrícola del vino, en la manufactura del corcho y en el proceso de empaque (cajas, cartones, papeles de etiquetado).

La llamada huella hídrica (punto 7 en la Tabla 4) corresponde al volumen total de agua dulce que se utiliza para consumo propio o para la producción de bienes o servicios; el producto vino en el caso de la Viña Modelo. Cuando se calcula la HdC de un producto, su huella hídrica debería incluir el consumo de agua de la empresa que fabrica el producto más el de todos sus suministradores, es decir, el consumo de agua en todo su ciclo de vida. En el caso particular de Viña Modelo, se utiliza el agua de pozo para el proceso agrícola del vino, donde se detectó la mayor parte del consumo de agua en la empresa. El resto corresponde a lo destinado al uso alimentario, sanitario y lavado, los cuales representan un porcentaje no significativo como aporte a la HdC.

El uso del suelo (punto 8 en la Tabla 4) se contabiliza como las hectáreas de suelo utilizado, ya sea sobre tierra firme o sobre agua, destinado a zonas de cultivo, jardines, entre otros. Se contabilizaron las 320 hectáreas de plantaciones de la viña junto con alrededor de una hectárea adicional estimada, que representa la planta de producción y los caminos construidos. La categoría de residuos, vertidos y emisiones (punto 9 de la Tabla 4) se clasifican según la tipología de los residuos: peligrosos, no peligrosos o radiactivos. Para el caso de Viña Modelo la única información obtenida es el gasto anual del tratado de los residuos, principalmente restos vegetales derivados de la recepción y despalillado de las uvas, los cuales son molidos y utilizados como compostaje para el cultivo de la viña.

La Tabla 7 resume los valores totales ingresados a la planilla del método MC3 para las diversas categorías discutidas arriba y se resaltan las principales observaciones sobre la aplicación específica a la Viña Modelo. Los contenidos de esta Tabla no son necesariamente todos los detallados en la Tabla 4 porque hay algunas categorías que no se aplican al caso específico en estudio. Por ejemplo en la categoría de materiales no aparecen las subcategorías de materiales amortizables genéricos y en obras y el uso de la infraestructura pública.

En la primera columna se presentan las categorías que aplican al caso en estudio mientras que en la segunda columna se muestran los valores usados, en las unidades requeridas por el método. En la tercera columna se presenta una serie de observaciones que explican la forma en que fue considerada dicha categoría y el valor asignado. Por ejemplo, en las emisiones directas se utiliza el poder calorífico del combustible real usado y luego se aplica un factor de emisión del combustible utilizado. La cuenta de electricidad se distribuye de acuerdo a los porcentajes de distribución de energía en Chile. Se explica también que en la categoría de materiales se realizó una conversión monetaria de euros a pesos chilenos y se establece el cambio y la fecha de la conversión. 
Tabla 7: Selección de datos utilizados para calcular la HdC de la Viña Modelo usando la metodología MC3

\begin{tabular}{|c|c|c|}
\hline Categorías & Datos & Observaciones sobre el método MC3 \\
\hline $\begin{array}{l}\text { 1. Emisiones directas: } \\
\text { 1.1. Combustible } \\
\text { Gasoil A (I) }\end{array}$ & $51.281,6$ & $\begin{array}{l}\text { Utiliza el poder calorífico del combustible } \\
\text { para convertir a unidades de GJ; luego } \\
\text { utiliza el factor de emisión del combustible } \\
\text { utilizado (según el inventario de GEI de } \\
\text { España) para obtener la huella emitida }\end{array}$ \\
\hline $\begin{array}{l}\text { 2. Emisiones indirectas: } \\
\text { 2.1. Electricidad } \\
\text { Térmica }(\mathrm{kWh}) \\
\text { Térmica de gas }(\mathrm{kWh}) \\
\text { Hidráulica }(\mathrm{kWh}) \\
\text { Eólica }(\mathrm{kWh}) \\
\text { Biomasa }(\mathrm{kWh})\end{array}$ & $\begin{array}{l}28.512 \\
81.446,4 \\
108.045,6 \\
1.626,7 \\
197,8\end{array}$ & $\begin{array}{l}\text { Se distribuye la cuenta de electricidad de } \\
\text { acuerdo a los porcentajes de distribución } \\
\text { energética de Chile. Luego se ingresan los } \\
\text { valores obtenidos a las celdas } \\
\text { correspondientes. }\end{array}$ \\
\hline $\begin{array}{l}\text { 3. Materiales: } \\
\text { 3.2. Materiales no amortizables } \\
\text { Derivados del vidrio }(\$) \\
\text { Abonos }(\$)\end{array}$ & $\begin{array}{l}3.448 .516 \\
1.728 .000\end{array}$ & $\begin{array}{l}\text { Se realizó previamente la conversión } \\
\text { monetaria de Euros a Pesos Chileno en la } \\
\text { pestaña complementaria "Aduana" } \\
\text { utilizando la conversión de Mayo } 2011 \\
(1 €=\$ 672) \text {. }\end{array}$ \\
\hline $\begin{array}{ll}\text { 4. } & \text { Servicios y contratas: } \\
\text { 4.1. } & \text { Baja movilidad } \\
& \text { Servicios externos de oficina }(\$) \\
& \text { Servicios de oficina de alto valor }(\$) \\
& \text { Servicios de hospedería }(\$) \\
& \text { Telefonía }(\$) \\
& \text { Formación externa }(\$) \\
& \text { Servicios interiores de limpieza }(\$) \\
\text { 4.2. } & \text { Alta movilidad } \\
& \text { Servicios exteriores de limpieza }(\$) \\
& \text { Correo, paquetería, mensajería }(\$) \\
\text { 4.3. } & \text { Transporte de personas } \\
& \text { Taxi }(\$) \\
& \text { Avión }(\$) \\
\text { 4.5. Uso de infraestructura pública } & \text { IVA declarado }(\$) \\
\text { Otros impuestos o tributos }(\$)\end{array}$ & $\begin{array}{l}22.174 .979 \\
2.994 .757 \\
6.386 .360 \\
9.335 .285 \\
15.000 \\
2.566 .898 \\
\\
3.629 .000 \\
1.034 .892 \\
\\
10.814 .513 \\
630.528 \\
\\
1.103 .196 \\
135.833 .277\end{array}$ & $\begin{array}{l}\text { Las cuentas contables son transformadas a } \\
\text { toneladas anuales de } \mathrm{CO}_{2} \text { usando los } \\
\text { factores de conversión generados en la } \\
\text { pestaña complementaria "Servicios". }\end{array}$ \\
\hline $\begin{array}{l}\text { 5. Recursos agrícolas y pesqueros: } \\
\text { 5.1. Vestuario y manufacturas } \\
\text { Vestuario y textil confeccionado de } \\
\text { algodón }(\$)\end{array}$ & 716.390 & $\begin{array}{l}\text { El procedimiento de cálculo está apoyado } \\
\text { en datos de la pestaña complementaria } \\
\text { "mat c" interviniendo las conversiones } \\
\text { monetarias previamente realizadas en } \\
\text { "Aduana". }\end{array}$ \\
\hline $\begin{array}{l}\text { 6. Recursos forestales: } \\
\text { Trozas de madera, puntales, etc. }(\$) \\
\text { Mobiliario con base madera }(\$) \\
\text { Productos editoriales }(\$) \\
\text { Manufacturas del corcho }(\$)\end{array}$ & $\begin{array}{l}892.350 \\
1.224 .000 \\
67.973 .696 \\
2.777 .530 \\
\end{array}$ & $\begin{array}{l}\text { Cálculo apoyado en la pestaña } \\
\text { complementaria "mat c". }\end{array}$ \\
\hline $\begin{array}{l}\text { 7. Agua: } \\
\text { 7.1. Consumo agua potable } \\
\text { Uso alimentario }(\$) \\
\text { Uso sanitario y lavado }(\$) \\
\text { 7.2. Consumo de agua no potable } \\
\text { Riego de jardines }(\$)\end{array}$ & $\begin{array}{l}346.200 \\
807.800 \\
4.807 .600\end{array}$ & $\begin{array}{l}\text { La mayor parte del agua utilizada en el } \\
\text { proceso es extraída de pozos en la } \\
\text { propiedad de la empresa, por lo que no } \\
\text { figuran dentro de las cuentas contables y } \\
\text { no se consideraron. }\end{array}$ \\
\hline $\begin{array}{l}\text { 8. Uso del suelo: } \\
\text { 8.1. Sobre tierra firme } \\
\text { Zonas de cultivo }(\mathrm{Ha})\end{array}$ & 321 & $\begin{array}{l}\text { Intervienen los factores de absorción de la } \\
\text { pestaña complementaria "in" }\end{array}$ \\
\hline 9. Residuos, vertidos y emisiones (\$) & 4.000 .000 & $\begin{array}{l}\text { En la viña Modelo la mayor parte de los } \\
\text { residuos son restos vegetales que se tratan } \\
\text { y reincorporan al campo como abono. }\end{array}$ \\
\hline
\end{tabular}




\section{RESULTADOS Y DISCUSIÓN}

Los resultados obtenidos por medio de la metodología MC3 son separados en resultados intermedios, para luego ser analizados por categorías. Asimismo, se comenta una serie de observaciones sobre el proceso de cálculo de la $\mathrm{HdC}$. Los resultados parciales (en ton $\mathrm{CO}_{2}$ por año) fueron obtenidos automáticamente al momento de ingresar la información correspondiente en la hoja de cálculo. La Planilla de cálculo usando la metodología MC3 está disponible en forma gratuita en la Internet: http://bit.ly/10w3Pvx

\section{Resultados parciales por categoría}

La categoría que más aporta a la HdC en Viña Modelo corresponde a los recursos forestales (260.3 ton. $\mathrm{CO}_{2}$ ), centrándose principalmente en materiales de empaque y etiquetado utilizados; el segundo mayor aporte procede de las emisiones directas por uso del petróleo diésel para las maquinarias industriales utilizadas en los procesos de producción del vino (132.2 ton. $\mathrm{CO}_{2}$ ); en tercer lugar se encuentra el aporte de las emisiones indirectas, especialmente por consumo de electricidad dentro de la organización (78.9 ton. $\mathrm{CO}_{2}$ ). La categoría de Servicios y Contratas aporta 39.1 ton. de $\mathrm{CO}_{2}$; el resto de las categorías presentan un aporte no relevante en el caso de Viña Modelo (menos del 1\%). La Tabla 8 lista la HdC parcial de cada categoría.

Tabla 8: Resultados por categoría en Viña Modelo

\begin{tabular}{|l|c|}
\hline Categorías de Consumo & $\mathrm{CO}_{2}$ Total [ton] \\
\hline Emisiones Directas & 132.2 \\
\hline Emisiones Indirectas & 78.9 \\
\hline Materiales & 45.5 \\
\hline Servicios y Contratas & 39.1 \\
\hline Recursos Agrícolas y Pesqueros & 6.0 \\
\hline Recursos Forestales & 260.3 \\
\hline Agua & 0 \\
\hline Uso Del Suelo & 2.0 \\
\hline Residuos & 1.0 \\
\hline
\end{tabular}

Dada la importancia de las emisiones procedentes de los recursos forestales, se determinó cuáles son los principales contribuyentes a la HdC en esta categoría. Los cuatro ítems que tienen incidencia sobre la HdC de esta categoría de consumo son: productos editoriales, trozos de madera, mobiliario a base de madera y manufactura del corcho. Dentro de estos se destacan los productos editoriales con 238 ton. de $\mathrm{CO}_{2}$, correspondiente al $91 \%$ de esta categoría. Estos productos corresponden a cartón y papeles impresos utilizado en empaque y etiquetado.

La categoría de emisiones directas (132.2 ton. $\mathrm{CO}_{2} ; 23 \%$ del total) corresponde a las emisiones por la quema del combustible diésel utilizado principalmente en las maquinarias industriales utilizadas en todo el proceso de producción de vino. Por otro lado, las emisiones indirectas con un aporte de 78.9 ton. $\mathrm{CO}_{2}$ (el $14 \%$ del total de la categoría) corresponden al consumo de electricidad, el cual posee subcategorías referidas a las distintas fuentes de generación de energía, aspecto que no depende directamente de la gestión de organización. Luego de sumar los resultados parciales se obtiene la HdC de Viña Modelo, correspondiente al aporte de todas las categorías de consumo, obteniendo 565 ton. de $\mathrm{CO}_{2}$. Como huella del producto se obtiene $195 \mathrm{gr}$. de $\mathrm{CO}_{2}$ por cada botella de $750 \mathrm{~cm}^{3}$ de vino, considerando una producción anual (período 2011) de aproximadamente 2.9 millones de botellas.

Las principales dificultades para la aplicación de la metodología MC3, se centran en el proceso del ingreso de los datos contables, debido a que los centros de costo que utiliza la empresa en la actualidad difieren de las categorías de consumo que presenta la hoja de cálculo de la metodología. Una vez clasificada la información de la empresa, se recopila información sobre aquellas categorías de consumo en las que sí interviene la empresa, pero que no existe un registro sobre los gastos que implican, como por ejemplo, el uso de agua extraída de pozos propios. En los cálculos se consideró factores de conversión genéricos proporcionados por la metodología y se convirtió unidades monetarias de euro a pesos chilenos, como se indicó antes $(1 €=\$ 672)$. En la parte de consumo de energía eléctrica el consumo energético total en pesos se separó según los porcentajes de aporte a la red eléctrica de las distintas fuentes de energía en Chile (mix eléctrico, mostrado en la Tabla 6). Estos valores separados se ingresaron directamente a la pestaña "HC" que tiene los espacios correspondientes al consumo para cada fuente de energía. No se utilizó la pestaña "mix" de la planilla, ya que ésta separa las fuentes de energía de los proveedores españoles de electricidad. 


\section{Comparación con datos de la literatura}

Aunque hay algunos estudios presentados en la literatura abierta es difícil hacer una comparación certera porque los métodos usados son distintos, las unidades son distintas, la información es incompleta o los alcances no están claros en cada caso. A pesar de ello hay algunos antecedentes que permiten hacer un análisis y comparación general.

Colman y Päster (2007) presentan un estudio donde analizan la HdC de varios vinos de distintas regiones del mundo. Para un vino francés de la región de la Loire encuentran $0.8 \mathrm{Kg} . \mathrm{CO}_{2} /$ botella mientras que un vino de Bordeaux da $2.0 \mathrm{Kg} . \mathrm{CO}_{2} /$ botella. Para un vino australiano de Yenda reportan $1.6 \mathrm{Kg}$. $\mathrm{CO}_{2} / \mathrm{botella} \mathrm{y}$ para un vino estadounidense de California indican $1.4 \mathrm{Kg} . \mathrm{CO}_{2} /$ botella. Para los distintos vinos los autores consideran distintas etapas del proceso. Para los vinos franceses consideran embotellado, producción y cultivo mientras que para el australiano y el californiano consideran embotellado, producción, cultivo, barril y suelo. Niccolucci et al. (2008) determinan la huella ecológica de dos vinos italianos de la zona de Toscana y consideran cuatro etapas principales del proceso: agricultura, viñedo, empaque y distribución. Estos autores determinaron la huella ecológica de un vino orgánico $\left(4.1 \mathrm{Kg} . \mathrm{CO}_{2} /\right.$ botella) y de un vino tradicional (8.13 $\mathrm{Kg}$. $\mathrm{CO}_{2}$ /botella). Además, los autores amortizan las emisiones de los materiales no consumibles y usan un factor de secuestración de 0.271 gha/ $\mathrm{COO}_{2}$. Una gha/tCO 2 (hectárea global por tonelada de $\mathrm{CO}_{2}$ ) corresponde a las hectáreas biológicamente productivas que pueden secuestrar una tonelada de $\mathrm{CO}_{2}$.

Soja et al. (2010) analizan la HdC de nueve empresas vitivinícolas del Valle de Traisen en Austria determinando un valor promedio de $1.73 \mathrm{Kg} . \mathrm{CO}_{2} /$ botella. Consideran cuatro etapas principales del proceso: viñedo (aporta 31\%), empaque (aporta 39\%), producción y guarda (aporta 17\%), transporte (aporta 12\%). Bosco et al. (2011) determinan la HdC de cuatro vinos italianos de diferentes características, producidos en la zona de Toscana usando el análisis de ciclo de vida. Encuentran que la HdC total de los vinos varía entre 0.6 y $1.3 \mathrm{Kg}$. $\mathrm{CO}_{2} /$ botella. La relación entre la producción anual de botellas y la HdC por botella para cada viña italiana estudiada fue de: i) $1.3 \mathrm{Kg} . \mathrm{CO}_{2}$ por botella para la viña que produce 23 mil botellas; ii) $1.1 \mathrm{Kg}$. CO2 para la que produce 39 mil; iii) $0.9 \mathrm{Kg} . \mathrm{CO}_{2}$ para la de $132 \mathrm{mil}$; y iv) $0.6 \mathrm{Kg} . \mathrm{CO}_{2}$ para la de 250 mil botellas. Estos números indican que no hay una relación directa entre huella y número de botellas producidas. Esto es porque hay producciones fijas que dependen poco de la cantidad producida. Cichelli et al. (2012) determinan la HdC con la calculadora de la HdC del Protocolo Internacional de Cálculo de la HdC a partir de un estudio previo de análisis del ciclo de vida (WI, 2012). El cálculo considera desde el viñedo hasta la venta al consumidor y no considera las emisiones del ciclo corto del carbono. Esto puede explicar el bajo valor reportado por los autores de $0.1 \mathrm{Kg} . \mathrm{CO}_{2}$ por botella producida.

Estos y otros casos aparecen detallados en la Tabla 9 en la que se presenta la HdC determinada para vinos de distinta procedencia, a fin de comparar los resultados obtenidos por diversos autores. Como se explicó antes y como se observa en la Tabla, los distintos autores consideran distintas etapas del proceso, en algunos casos se reportan valores globales y no parciales, en la mayoría de los casos los alcances no están claramente definidos y los volúmenes de producción no son siempre conocidos. Esto hace difícil, como se mencionó más arriba, hacer una comparación certera entre la HdC de la Viña Modelo y otras para determinar cómo está operando la planta desde el punto de vista medioambiental. En aquellos casos en que los autores consideran categorías adicionales a las de este trabajo, sólo se consideró las categorías comunes para efectos de comparación. Para los casos en que los autores entregan el resultado en alguna unidad de medida diferente, se utilizaron factores de conversión genéricos para utilizar una unidad común, $\mathrm{kg} \mathrm{CO} /$ botella.

Para contextualizar estos números y su significado con respecto a la emisión de GEl en vitivinícolas y en otras industrias se incluyen algunos resultados para la HdC de otros productos. Iribarren et al. (2010), por ejemplo, determinan la HdC de almejas envasadas usando la metodología PAS 2050 mientras que Avraamides y Fatta. (2008) cuantifican la huella para aceite de oliva usando el análisis del ciclo de vida e incluyendo todas las posibles fuentes de GEI. Rotz et al. (2010) y también Flysjö et al. (2011) determinan la $\mathrm{HdC}$ de leche procesada usando diferentes métodos y obteniendo también resultados diferentes. Estos números permiten formarse una idea general del significado de los valores de la HdC para vinos en comparación con otros productos del mercado.

\section{ADOPCIÓN DE LA METODOLOGÍA MC3}

La adopción del concepto de la HdC como determinación de cuán amigable con el medio ambiente ha sido el proceso de elaboración de un producto requiere de una serie de cambios en todos los niveles de la organización. Por lo tanto, el proceso de adopción de una metodología para cuantificar la HdC requiere de una especial disposición de la empresa y entonces el método a aplicar adquiere especial importancia. Probablemente el punto de partida para una exitosa adopción del concepto y para la cuantificación de la 
$\mathrm{HdC}$ es el convencimiento de la empresa de que la $\mathrm{HdC}$ constituye un factor de competitividad importante, en especial en el mercado internacional que inevitablemente impondrá restricciones basadas en producción sustentable (Schneider y Samaniego, 2009). La simplicidad relativa del método MC3 sin duda facilita el proceso de adopción y su aplicación, sin demandar gastos apreciables al proceso contable de la empresa. Con el fin de implementar exitosamente estos cambios se recomienda tomar en cuenta los siguientes tres pasos: comunicación, cambio y confirmación.

Tabla 9: Resultados de algunos estudios sobre determinación de la $\mathrm{HdC}$ en vinos y otros productos comestibles. En la Tabla, la $\mathrm{HdC}$ de vinos está en $\mathrm{Kg}$. $\mathrm{CO}_{2}$ por cada botella de $0.75 \mathrm{~L}$.

\begin{tabular}{|c|c|c|c|c|}
\hline$N^{0}$ & Referencia & Qué se mide & $\mathrm{HdC} \mathrm{kg} \mathrm{CO} /$ botella & Comentarios \\
\hline 1 & $\begin{array}{l}\text { Colman y Päster } \\
(2007)\end{array}$ & $\begin{array}{l}\text { HdC utilizando análisis } \\
\text { del ciclo de vida }\end{array}$ & $\begin{array}{l}\text { Vino francés } 1=0.8 \\
\text { Vino francés } 2=2.0 \\
\text { Vino australiano }=1.6 \\
\text { Vino USA }=1.4\end{array}$ & $\begin{array}{l}\text { Para los distintos vinos los autores } \\
\text { consideran distintas etapas del proceso. } \\
\text { Gran parte de la discusión se centra en el } \\
\text { transporte hasta el consumidor final. }\end{array}$ \\
\hline 2 & Niccolucci et al. (2008) & $\begin{array}{l}\text { Huella ecológica de } \\
\text { vinos italianos } \\
\text { orgánico y tradicional }\end{array}$ & $\begin{array}{l}\text { Orgánico = } 1.4 \\
\text { Tradicional = } 2.5\end{array}$ & $\begin{array}{l}\text { Considera cuatro etapas: agricultura, } \\
\text { viñedo, empaque y distribución, de los } \\
\text { cuales la principal corresponde a } \\
\text { agricultura, con un aporte del } 67 \% \text { para lo } \\
\text { orgánico y } 76 \% \text { para lo tradicional. }\end{array}$ \\
\hline 3 & Soja G. et al. (2010) & $\begin{array}{l}\text { HdC de nueve } \\
\text { empresas vitivinícolas } \\
\text { del Valle Traisen de } \\
\text { Austria }\end{array}$ & Vino austriaco $=1.7$ & $\begin{array}{l}\text { Considera } 4 \text { etapas: viñedo (31\%), } \\
\text { empaque (39\%), producción y guarda } \\
(17 \%) \text {, transporte }(12 \%) .\end{array}$ \\
\hline 4 & Bosco et al. (2011) & $\begin{array}{l}\text { HdC usando análisis } \\
\text { del ciclo de vida }\end{array}$ & $\begin{array}{l}\text { Italiano } 1=1.0 \\
\text { Italiano } 2=0.8 \\
\text { Italiano } 3=0.8 \\
\text { Italiano } 4=0.5\end{array}$ & $\begin{array}{l}\text { Se estudian cuatro viñedos de diferentes } \\
\text { características y el resultado aparece } \\
\text { disgregado por etapa del proceso. }\end{array}$ \\
\hline 5 & Cichelli et al. (2012) & $\begin{array}{l}\text { HdC con el calculador } \\
\text { de la huella del } \\
\text { carbono (IWCC) }\end{array}$ & Italiano = 0.1 & $\begin{array}{l}\text { El estudio parte de un estudio previo de } \\
\text { análisis del ciclo de vida y considera desde } \\
\text { el viñedo hasta la venta al consumidor. }\end{array}$ \\
\hline 7 & Carballo et al (2009) & $\begin{array}{l}\text { HdC usando MC3 } \\
\text { para un vino español }\end{array}$ & Vino español= 3.8 & $\begin{array}{l}\text { Las emisiones se concentran en I } \\
\text { categoría materiales no amortizables con } \\
\text { un } 67 \% \text { (principalmente por los químicos y } \\
\text { derivados de vidrio utilizado), y un } 12 \% \\
\text { corresponde a los recursos forestales. }\end{array}$ \\
\hline 7 & Viña Anakena (2011) & $\begin{array}{l}\text { HdC corporativa para } \\
\text { el año } 2009\end{array}$ & Vino chileno=2.0 & $\begin{array}{l}\text { se usó la técnica definida por el Instituto } \\
\text { para la Reducción del Carbón (CRI) } \\
\text { según los límites fijados en el programa } \\
\text { Carbon Neutral. }\end{array}$ \\
\hline 11 & Iribarren et al. (2010) & $\begin{array}{l}\text { HdC de almejas } \\
\text { envasadas }\end{array}$ & $\begin{array}{l}\text { Almejas }=1.4 \\
\text { (Kg. } \mathrm{CO}_{2} / \text { tarro de } 200 \\
\text { gr.) }\end{array}$ & $\begin{array}{l}\text { Utiliza la metodología PAS } 2050 \text { identifican } \\
\text { el envasado y el manejo de las conchas } \\
\text { (residuos) como las principales causantes } \\
\text { de la alta HdC. }\end{array}$ \\
\hline 12 & $\begin{array}{l}\text { Avraamides y Fatta } \\
(2008)\end{array}$ & HdC de aceite de oliva & $\begin{array}{l}\text { Aceite }=4.9 \\
\mathrm{Kg} . \mathrm{CO}_{2} / \mathrm{L} \text { aceite }\end{array}$ & $\begin{array}{l}\text { Usan el análisis del ciclo de vida para } \\
\text { determinar la HdC por litro de aceite en } \\
\text { Chipre, incluyendo todas las posibles } \\
\text { fuentes de GEI. }\end{array}$ \\
\hline 13 & Rotz et al. (2010) & $\begin{array}{l}\text { HdC de leche } \\
\text { procesada }\end{array}$ & $\begin{array}{l}\text { Leche }=0.4-0.7 \\
\left(\mathrm{Kg} . \mathrm{CO}_{2} / \mathrm{Kg} \cdot \mathrm{de}\right. \\
\text { leche) }\end{array}$ & $\begin{array}{l}\text { Se usa un ciclo de vida parcial y no se } \\
\text { considera el ciclo corto del Carbono. Los } \\
\text { GEl estimados incluyen } \mathrm{CO}_{2}, \mathrm{CH}_{4} \text { y } \mathrm{N}_{2} \mathrm{O} \text {. }\end{array}$ \\
\hline 14 & Flysjö et al. (2011) & $\begin{array}{l}\text { HdC de leche } \\
\text { procesada }\end{array}$ & $\begin{array}{l}\text { Suecia }=1.2 \\
\text { Nueva Zelanda }=1.0 \\
\left(\mathrm{Kg} . \mathrm{CO}_{2} / \mathrm{L} \text { de leche }\right)\end{array}$ & $\begin{array}{l}\text { Se realiza un análisis hasta la puerta de la } \\
\text { granja (sin despacho), se compara una } \\
\text { granja al aire libre en Nueva Zelanda y una } \\
\text { interior en Suecia. }\end{array}$ \\
\hline 15 & Este trabajo & $\begin{array}{l}\text { HdC usando MC3 } \\
\text { para una vitivinícola } \\
\text { en Chile }\end{array}$ & Vino chileno $=0.2$ & $\begin{array}{l}\text { Las contribuciones parciales se concen- } \\
\text { tran un } 46 \% \text { en los recursos forestales y } \\
\text { un } 23 \% \text { en las emisiones directas por el } \\
\text { uso del combustible diésel. }\end{array}$ \\
\hline
\end{tabular}


La comunicación corresponde al primer paso en el proceso de adopción y es la etapa que sienta bases para la continuidad y sustento del cambio a implementar. Este acto debe involucrar a todo el personal de la empresa, presentando argumentos sólidos para su implementación, con el fin de crear una visión clara y compartida en toda la organización. Es de suma importancia esta primera etapa, debido a que implica compromiso y motivación de todo el personal para aceptar los cambios.

Una vez comunicada la intención de adoptar el cambio se procede a ejecutar el plan de trabajo reuniendo todos los elementos necesarios, sean éstos físicos o intelectuales, para comenzar a efectuar las acciones pertinentes a cada nivel organizacional, evaluando cada etapa y generando acciones correctivas en caso que fuese necesario. Al momento en que la adopción se haya integrado efectivamente a cada nivel de la organización se asume como una práctica habitual dentro de la empresa, creando políticas que apoyen, sostengan y confirmen dicha adopción.

Cada una de las etapas en el proceso de adopción requiere también de una serie de actividades paralelas para la preparación del personal y la alineación de los trabajadores a la nueva visión de la organización. La empresa debe también hacer los cambios tecnológicos necesarios en los sistemas y procesos productivos, convencida de que se trata de inversiones que en el mediano y largo plazo representarán ingresos adicionales o la sustentabilidad misma de la empresa.

\section{CONCLUSIONES}

Del estudio realizado se pueden deducir varias conclusiones relacionadas con el tema de la $\mathrm{HdC}$, con el método usado, con el proceso de adopción y con los factores más influyentes en la determinación de la $\mathrm{HdC}$.

1.- La metodología MC3 resulta adecuada para ser adoptada en Viña Modelo por su bajo costos de implementación, por su relativa facilidad de comprensión y aplicación, y por la disponibilidad de la información requerida por el método.

2.- El proceso para obtener la información necesaria tuvo un grado menor de dificultad, considerando los tipos de registros actuales que dispone el proceso de producción y gestión en Viña Modelo y que son distintos a los requeridos por el método MC3.

3.- La adopción de una metodología depende principalmente del interés de la empresa, por lo que el costo de su proceso es uno de los principales aspectos para que se tome la decisión de adoptar un método de estimación de la HdC.

4.- El factor más influyente dentro de las fuentes controladas por la empresa corresponde a las emisiones por parte de las maquinarias del proceso productivo del vino, representando más de la mitad de la emisión total del producto.

5.- El método MC3 indica, desde un enfoque corporativo, que el elemento que más contribuye al valor de la HdC del producto son los recursos forestales, representando el $46 \%$ de la emisión total de la organización.

\section{REFERENCIAS}

ABC, Association Bilan Carbone. Disponible en http://bit.ly/VtvQ3C Acceso en dic. (2012)

Arias, L.; Arruez, E. Estimación de la huella de carbono mediante metodología MC3 en el sector construcción, Memoria de Titulación del Dpto. de Ingeniería Industrial, Univ. de La Serena Chile (2011)

Avraamides, M.; Fatta D. Resource consumption and emissions from olive oil production: a life cycle inventory case study in Cyprus. Journal of Cleaner Production 16 (7) 809-821 (2008)

Bolin B.; Jäger J.; Döös B. The greenhouse effect, climatic change, and ecosystems. París (Francia): Scientific Commitee on Problems of the Environment (1986)

Borregaard N.; Medina J.; Carretero E.; Bordey E. Eficiencia energética y cambio climático en el sector vitivinícola: procesos, herramientas y ejemplos de buenas prácticas. Santiago de Chile: Proyecto del Nodo Tecnológico "Energía y cambio climático" Disponible en http://bit.ly/XZ3PkO Acceso en may. 2012 (2009)

Bosco S.; Di Bene C.; Galli M.; Remorini D.; Massai R.; Bonari E. Greenhouse gas emissions in the agricultural phase of wine production in the Maremma rural district in Tuscany, Italy. Italian J. Agronomy, 6(15) 93-100 (2011)

BSI, British Standards Institution. PAS 2050: 2011, Specification for the assessment of the life cycle greenhouse gas emissions of goods and services. London: BSI Group (2011) 
Cichelli A.; Raggi A.; Pattara C. Life cycle assessment and carbon footprint in the wine supply-chain. Environmental Management, 49(6) 1247-58 (2012)

Colman T.; Päster P. Red, white and green: the cost of carbon in the global wine trade. Disponible en http://bit.ly/Xugihi Acceso en ene. 2013 (2007)

Carballo A.; García-Negro M.; Dómenech J.L. El MC3 una alternativa metodológica para estimar la huella corporativa del carbono (HCC). Revista Desarrollo Local Sostenible, 2(5) 10-16. (2009)

Dómenech J.L. Huella ecológica portuaria y desarrollo sostenible. Puertos, 114, $26-31$ (2004).

Dómenech J.L. Estándares 2010 de huella de carbono MC3, Gijón, España: CONAMA 10. (2010)

Espíndola C.; Valderrama J.O., Huella del carbono. Parte 1: conceptos, métodos de estimación y complejidades metodológicas. Información Tecnológica, 23(1) 163-176. (2012a)

Espíndola C.; Valderrama J.O., Huella del carbono. Parte 2: la visión de las empresas, los cuestionamientos y el futuro. Información Tecnológica, 23(1) 177-192. (2012b)

Flint D.; Golicic S. L. Searching for competitive advantage through sustainability: A qualitative study in the New Zealand wine industry. International Journal of Physical Distribution and Logistics Management, 39(10), 841-860 (2009)

Flysjö A.; Cederberg C.; Henriksson M.; Ledgard S. How does co-product handling affect the Carbon Footprint of milk? - Case study of milk production in New Zealand and Sweden. Int. J. Life Cycle Assess. 16, 420-430 (2011)

GEI, GHG Protocol. The Greenhouse Gas Protocol, a corporate accounting and reporting standard. Disponible en http://bit.ly/ukwepr Acceso en abr. (2011).

Iribarren D.; Hospido A.; Moreira M.; Feijoo G. Carbon footprint of canned mussels from a business-toconsumer approach. A starting point for mussel processors and policy makers. Environmental science and policy 13 (6) 509-521 (2010)

Niccolucci V.; Galli A.; Kitzes J.; Pulselli R.M.; Borsa S.; Marchettini N. Ecological Footprint analysis applied to the production of two Italian wines. Agriculture, Ecosystems and Environment (128), 162-166. (2008)

Peters G.; Hertwich E. $\mathrm{CO}_{2}$ Embodied in international trade with implications for global climate policy. Environ. Sci. Technol. 42(5) 1401-1407. (2008)

Rotz C.A.; Monte F.; Chianese D.S. The carbon footprint of dairy production systems through partial life cycle assessment. Journal of dairy science 93 (3), 1266-1282 (2010)

Schneider H.; Samaniego J. La huella del carbono en la producción, distribución y consumo de bienes y servicios, Santiago de Chile: documentos de proyectos (2009)

Sirieix L.; Remaud H. Consumer perceptions of eco-friendly vs. conventional wines in Australia. $5^{\circ}$ Conferencia International Academy of Wine Business Research. Auckland, Nueva Zelanda (2010)

Smyth M.; Russell J.; From graft to bottle. Analysis of energy use in viticulture and wine production and the potential for solar renewable technologies. Renewable and Sustainable Energy Reviews 13 (8), 1985-1993 (2009)

Soja G.; Zehetner F.; Rampazzo-Todorovic G.; Schildberger B.; Hackld K.; Hofmann R.; Burger E.; Omann I.; Wine production under climate change conditions: mitigation and adaptation options from the vineyard to the sales booth. $9^{\circ}$ European IFSA Symposium. Vienna, Austria (2010)

Thompson D.; Forbes S. L. Going "green" to find "gold" in wine: A case study of a sustainable New Zealand wine producer. Bourdeaux, Bordeaux Management School (2011)

Thompson K. Wine Packaging Alternatives. Not All Good Wine Comes in Glass Bottles. Investigation of the Department of Food Science and Technology of Virgnia Polytechnic Institute And State University, Blacksburg, Virginia USA (2010)

Valderrama J.O.; Espíndola C.; Quezada R. La Huella de Carbono, un concepto que no puede estar ausente en cursos de ingeniería y ciencia. Formación Universitaria 4 (3), 3-12. (2011)

Viña Anakena. Reporte de Sustentabilidad. Disponible en http://bit.ly/14vF1Xa. Acceso ene. 2013 (2011)

Waye V. Carbon footprints, food miles and the Australian wine industry. Melb. J. Int'I L. 9, 271. Disponible en http://bit.ly/wdpd3g Acceso en dic. 2012 (2008)

WI, Wine Institute, California-USA, http://www.wineinstitute.org. Acceso en dic. (2012) 